\title{
Trends of Seroprevalence, Epidemiology and Clinical Presentation of HIV in North India: A Tertiary Care Hospital Based Study
}

\author{
Varun Goel*, Dinesh Kumar, Vrushali Patwardhan, Veena Balooni, Shalini Singhal, \\ Sarman Singh \\ Clinical Microbiology and Molecular Medicine, Department of Laboratory Medicine, \\ All India Institute of Medical Sciences (AIIMS), New Delhi, India \\ Email: *drvarun21@gmail.com
}

Received 26 March 2016; accepted 5 June 2016; published 8 June 2016

Copyright (C) 2016 by authors and Scientific Research Publishing Inc.

This work is licensed under the Creative Commons Attribution International License (CC BY). http://creativecommons.org/licenses/by/4.0/

(c) (i) Open Access

\begin{abstract}
Introduction: HIV infection represents a major public health problem for both developing and developed countries as it has grown to global pandemic. Spectrum of clinical presentation of HIV can greatly vary with geopolitical, socioeconomic and cultural environment. Aims: The aim of this study was to estimate the prevalence, socio-demographic conditions, clinical presentations, opportunistic infections and the possible associated risk factors for acquiring HIV infection. Materials and Methods: An observational prospective study was conducted from January 2010 to December 2014 at our centre situated in north India. As per the strategy and policy prescribed by NACO, tests were performed on the serum samples. Results: Out of the total 35369 clients tested for HIV infection, 292 were found to be HIV-1 seropositive. Two HIV-2 cases were found among the studied population. The seroprevalence of HIV was found to be $0.83 \%(294 / 35369)$. Mean age of the study group was $30 \pm 5.65$ (range: $02-80$ ) years. Overall positivity rates among attendees were found to be $0.97 \%(138 / 14098), 0.96 \%(35 / 3610), 0.82 \%(7 / 850), 0.78 \%(22 / 2810)$ and $0.65 \%(92 / 14001)$ in the years $2010,2011,2012,2013$ and 2014 respectively. Heterosexual route of transmission was the major route of infection in $78.2 \%$ patients. At the time of presentation, it was observed that Tuberculosis $(14.9 \%)$ was the most common opportunistic infection. Conclusion: The study shows that there is a decreasing trend in the HIV prevalence in North India. However, the number of HIV cases is still significant and suggests the need for focused prevention efforts in high-risk groups.
\end{abstract}

\section{Keywords}

AIDS, Clinico-Epidemiological Profile, Heterosexual, HIV, Prevalence, India

\footnotetext{
${ }^{*}$ Corresponding author.
} 


\section{Introduction}

Among all other medical conditions, HIV has been the greatest social enigma and has shown its impact on the global economy. India has the third largest number of people living with HIV/AIDS in the world with a total number of people living with HIV (PLHIV) estimated at 21.17 lakhs in 2015 [1] [2]. According to the National AIDS Control Organization (NACO), the HIV prevalence among adults at the national level has continued its steady decline from $0.38 \%$ in 2001-03 to 0.26\% in 2015 [2]. Between 2000 and 2015, new HIV infections dropped from 2.51 lakhs to 86 thousand in India, a reduction of 66\% against a global average of 35\% [3]. Delhi is a low prevalent state but is highly vulnerable due to a large number of high risk population, migrants and long distant truck drivers. The clinical course of the disease with HIV and pattern of opportunistic infections vary from country to country and also from patient to patient. In tropical countries like India, a handful of pathogens cause most of the opportunistic infections [4]. HIV counseling and testing services are a key entry point to HIV prevention and care of people who are infected with HIV.

There is a need to know the profile of patients with HIV and characterize their clinical and socio-demographic variables in the management. Moreover, there is need to understand the presentation of HIV disease in the local context and cultural environment. The present study is aimed to estimate the prevalence of HIV infection, sociodemographic conditions, clinical presentations and the possible associated risk factors for acquiring HIV infection at a tertiary care hospital in North India.

\section{Materials and Methods}

The present observational prospective study was carried out over a period of five years from January 2010 to December 2014. At this Institute we use a modified client and time friendly method, in which HIV/AIDS educative posters are displayed on various patient counters so that while the client waits for his turn for consultation or sample receiving, he/she also can read salient features of various tests and about HIV/AIDS [5]. The protocol of the rapid tests performed as per NACO guidelines was as follows: The first test kit was of highest sensitivity and the specificity increases with second and third kit. Therefore, when the test with first test kit is negative, second and third kit tests were not done and the result is negative for that tested sample. If the first test is positive, then second and third rapid kit tests were performed and if these two tests were also positive, then the final result was given as positive for that tested sample [6]. At least one of the above-mentioned tests should be able to differentiate between HIV-1 and HIV-2 according to the guidelines. The samples which were positive for HIV-2 infection were confirmed by Western blot assay for the detection of HIV antibodies in human serum/plasma following manufacturer's instructions. Only HIV positive patients are post-test counseled in detail [5] [7]. Confidentiality of collected data was ensured to the participants. The clients were approached by the investigator, and informed consent was obtained for inclusion in the study. Those who gave consent for the study were interviewed with a structured pretested questionnaire to collate socio-demographic details: age, gender, education, occupation, socioeconomic status, demographic characteristics, marital status, sexual behavior, substance abuse including intravenous drug use (IDU). HIV infected cases were referred to antiretroviral therapy (ART) centre of our hospital for further management [7].

Statistical analysis: Statistical data analysis was done using the Statistical Package for Social Science software (SPSS version 20.0, IBM Corp., Armonk, NY) and Microsoft Office Excel 2010. Tests of significance were conducted using the chi-square test at a significance level of 0.05 .

\section{Results}

Over a period of five year of the 35369 clients screened for HIV, 292 tested positive for HIV-1. Two HIV-2 cases were found among the population studied from the year 2010 to 2014. No HIV-1/2 dual infection case was found among the population studied. In this study the seroprevalence of HIV was found to be 0.83\% (294/35369). Mean age of the study group was $30 \pm 5.65$ (range: 02 - 80) years. Overall positivity rates among attendees were found to be $0.97 \%$ (138/14098), $0.96 \%$ (35/3610), 0.82\% (7/850), 0.78\% (22/2810) and 0.65\% (92/14001) in the years 2010, 2011, 2012, 2013 and 2014 respectively. This decrease in the seroprevalence from $0.97 \%$ in 2010 to $0.65 \%$ during 2014 was statistically significant $(P=0.001$, confidence interval $[\mathrm{CI}]=95 \%)$. Females constituted more than one fourth of the study group (29.9\%).There was also a decrease in the percentage of female patients being tested from $0.72 \%$ (42/5793) in the year 2010 to $0.42 \%$ (24/5690) in 2014. Details of demographic characteristics and transmission categories are presented in [Table 1]. Maximum number of clients tested seropositive in the age 
Table 1. Demographic characteristics and transmission-risk categories in a series of 294 patients with HIV/AIDS.

\begin{tabular}{cc}
\hline Parameter & Number of patients, n (\%) \\
\hline Age, in years (mean \pm SD) & $30 \pm 5.65$ \\
Males & $206(70.1)$ \\
Outside Delhi & $95(32.3)$ \\
HIV-1 & $292(99.3)$ \\
HIV-2 & $2(0.7)$ \\
Occupation & \\
Laborer & $103(35)$ \\
Housewife & $53(18)$ \\
Driver & $41(13.9)$ \\
Professional & $19(6.5)$ \\
Others & $78(26.5)$ \\
Risk categories & \\
Heterosexual contact & $230(78.2)$ \\
Blood transfusion & $14(4.8)$ \\
Homosexual contact & $12(4.1)$ \\
Mnknown (patients denied any of the known risk factor) & $9(3.1)$ \\
\hline & $6(2.0)$ \\
\hline & $23(7.8)$ \\
\hline
\end{tabular}

group of 26 - 45 years (52.1\%), followed by 19.1\%, 18.4\%, 8.8\% and 1.7\% in the age groups of 12 - 25 years, 46 65 years, less than 12 years and above 65 years. In present study children less than 15 years of age accounted for $12.2 \%$ of all the HIV infections. Of the two HIV-2 cases both were males in the age group of 26 - 45 years. $59.9 \%$ of the clients had received primary education, $27 \%$ received up to higher secondary education, and $9.6 \%(n=18)$ were uneducated. Almost two-third of the clients (69\%) were married.

Laborers constituted 35\% of cases, $18 \%$ were housewife while $13.9 \%$ were truck drivers. Educated professionals were the least common occupational group. Most of the cases were from the lower middle and lower socio-economic classes. Unprotected heterosexual contact has come out to be the most common mode of transmission of HIV in the present study with the rate of $78.2 \%$ with the male and female ratio of 2.1:1. Transmission route for both the HIV-2 cases were through heterosexual contact with one case being of West African origin. Other transmission modes were mother-to-child transmission (MTCT) 14 (4.8\%), blood transfusion 12 (4.1\%), homosexual contact 9 (3.1\%) and injectable drug users (IDU) 6(2.0\%). Most of the men who have sex with men (MSM) were in age group of 26 - 45 years, school-level educated urban residents and were not involved in heterosexual activity. 7.8\% of clients did not gave history of any of the known risk factors for the acquisition of HIV infection.

A large number of cases (32.3\%) were from states other than Delhi. The common clinical presentations were fever (43.2\%), weight loss (41.4\%), diarrhea (34.6\%), cough (33.7\%) and primary generalized lymphadenopathy (8\%). At the time of presentation, we observed that Tuberculosis 44 (14.9\%) was the most common opportunistic infection(OI), followed by Candida (5.1\%), Herpes simplex virus (1.4\%), Cryptococcus neoformans (1.0\%) and Cryptosporidium parvum (1.0\%). Toxoplasma gondii, Leishmania donovani and Pneumocystis jirovecii were found in one case each.

\section{Discussion}

The present study highlights the seroprevalence and epidemiological data related to HIV infection from the Northern India in a tertiary care hospital. Present study showed overall seropositivity of $0.83 \%$ among patients. 
This is high as compared to the seroprevalence among the general population 0.35\% in 2014 [2]. Overall in this study a declining trend in HIV prevalence was seen from 2010 to 2014. This in contrast to some studies which showed rising trends in adult HIV prevalence in Delhi [8]. This may be due to the fact that this institution being a apex tertiary care centre caters not only to Delhi but also to all of the neighboring States and national capital region (NCR).

In contrast to the pandemic spread of HIV-1, the HIV-2 epidemic has largely been limited to West Africa. In present study $0.7 \%$ cases were reactive for HIV-2 which is in accordance to other studies which confirm that HIV-2 is relatively rare in Asia [9].

Most of the patients were males in the age group 26 - 45 years. This is similar to NACO nation-level statistics with an estimated adult (15 - 49 age group) HIV prevalence of $0.27 \%$ in 2011 [8]. This age group population is more affected because they are sexually more active with high numbers of sexual partners. In present study children less than 15 years of age accounted for $12.2 \%$ of all the HIV infections in contrast to $7 \%$ (1.45 lakh) of all infections in 2011 in India [8]. The male preponderance observed in present study might have been due to fact that females face more stigma and discrimination in a variety of settings including households, the community and workplaces so do not seek medical care fearing ostracism, loss of family support and financial constraint. Majority of the HIV seropositives belong to the married group irrespective of their sexes. Most of the patients were from the lower middle and lower socio-economic classes. Laborers constituted 35\% of patients while 13.9\% were truck drivers; $18 \%$ were housewife. Studies in the India have found high HIV (2\% - 14\%) among long distance truck drivers [10].

Heterosexual contact was the commonest mode of transmission in 230 (78.2\%) cases. This is similar with that reported by Lal et al. (84\%) in India [11]. HIV prevalence trend has witnessed significant decline among antenatal clinic attendees considered proxy for general population (0.49\% in 2007 to 0.35\% in 2012-2013), Female Sex Workers (5.06\% in 2007 to 2.67\% in 2011) and MSM (7.41\% in 2007 to $4.43 \%$ in 2011), and stable trends have been recorded among IDU (7.23\% in 2007 to $7.14 \%$ in 2011) at the national level [8]. In this study, the perinatal transmission was found to be 14 (4.8\%). Mother to child transmission may be low in present study since there is separate ICTC centre for screening the antenatal mothers in our hospital. In present study, only $4.1 \%$ patients gave the history of transmission through blood or blood products but it could not be verified that transmission was surely through blood products. IDU constituted only a minority of the study group (2.0\%) as has been observed in other parts of India except for the north-eastern states where IDU is widely prevalent [12]. History suggestive of a risk factor for HIV transmission could not be elicited in 23 (7.8\%) patients. Overall, 3.1\% of all HIV positive cases reported homosexual activity during the study period. Due to their diverse sexual mixing pattern, poor access to awareness programs and less utilization of control measures, MSM in India are at higher risk of sexually transmitted infections including HIV [13] [14].

Tuberculosis was the commonest OI in this study. Co-infection with HIV and TB has already been reported which is also one of the most significant global public health concerns [15]. In the present study, the HIV-TB co-infection rate was found to be $14.9 \%$ over the period of five years. Herpes simplex virus was present in 4 cases in this study. As such clinically confirmed Kaposi Sarcoma cases are rarely reported from India in spite of the high prevalence of the HHV-8 infection of around 26.0\% when compared with African nations and this is also likely to be responsible for the rarity of Kaposi's sarcoma [16]. Pneumocystis pneumonia was seen in only in one patient despite been reported to be the third commonest OI in southeast Asia and is known to be highly prevalent in the north-east Indian region [17].

The study shows that there is a decreasing trend in the HIV prevalence in North India. The results may not reflect the true prevalence of HIV in the community as this was a hospital-based study and patients coming to our tertiary care center represent just the tip of this iceberg. Also, the data reported here could be biased due to recall error and social stigma faced by the patients in the community. However inspite of these limitations, the number of HIV cases is still significant and health care personnel cannot be complacent with this trend. There is a male preponderance over female among sexually active age group of 26 - 45 years of age. Hence development of the appropriate policies and strategies like education, promoting safe behaviors, counseling, and awareness should focus on this age group which would help to bring down the number of AIDS cases.

\section{References}

[1] Joint United Nations Program on HIV/AIDS 2014. The Gap Report. Geneva. 
[2] National Institute of Medical Statistics and National AIDS Control Organization 2015 Technical Report India: HIV Estimates-2015. New Delhi.

[3] UNAIDS (2015) How AIDS Changed Everything.

[4] Gupta, V., Singla, N., Lehl, S.S. and Chander, J. (2007) Clinico-Epidemiological Profile of HIV Infection over a Period of Six Years in a North Indian Tertiary Care Hospital. Indian Journal of Medical Microbiology, 25, 171. http://dx.doi.org/10.4103/0255-0857.32732

[5] Singh, S. (Response), Read, J. and Winceslaus, S.J. (2003) New Strategies for Increasing the Detection of HIV: Analysis of Routine Data. BMJ, 326, 1066-1067. http://dx.doi.org/10.1136/bmj.326.7398.1066

[6] Singh, S., Ed. (1999) Standard Operative Manual: Hospital Infection Control. National AIDS Control Organization, Government of India, New Delhi.

[7] National AIDS Control Organization, Ministry of Health and Family Welfare, Government of India. Detection of HIV infection. In: Guidelines on HIV Testing, March 2007, 38-53.

[8] Annual Report 2013-14. National AIDS Control Organization. Department of AIDS Control. Ministry of Health \& Family Welfare, Government of India. New Delhi 2014. http://www.naco.gov.in/upload/2014\%20mslns/NACO_English\%202013-14.pdf

[9] Kashyap, B., Gautam, H. and Bhalla, P. (2011) Epidemiology and Seroprevalence of Human Immunodeficiency Virus Type 2. Intervirology, 54,151-155. http://dx.doi.org/10.1159/000319929

[10] Pandey, A., Mishra, R.M., Sahu, D., Benara, S.K., Biswas, M., Sengupta, U., et al. (2012) Heterosexual Risk Behaviour among Long Distance Truck Drivers in India: Role of Marital Status. Indian Journal of Medical Research, 136, 44-53.

[11] Lal, S. (2003) Surveillance of HIV/AIDS in India (Editorial). Indian Journal of Community Medicine, 27, 3-9.

[12] Mishra, R.K., Ganju, D., Ramesh, S., Lalmuanpuii, M., Biangtung, L., Humtsoe, C., et al. (2014) HIV Risk Behaviours of Male Injecting Drug Users and Associated Non-Condom Use with Regular Female Sexual Partners in North-East India. Harm Reduction Journal, 11, 5. http://dx.doi.org/10.1186/1477-7517-11-5

[13] Jha, U.M., Raj, Y., Venkatesh, S., Dhingra, N., Paranjpe, R.S. and Saggurti, N. (2014) HIV Epidemic among Men Who Have Sex with Men in India: National Scenario of an Unfinished Agenda. HIV AIDS (Auckl), 6, 159-170.

[14] HIV Sentinel Surveillance 2010-11. A Technical Brief. National AIDS Control Organization. Department of AIDS Control. Ministry of Health \& Family Welfare, Government of India. New Delhi. 2011. http://naco.gov.in/upload/Surveillance/Reports\%20\&\%20Publication/HSS\%202010-11 Technical\%20Brief 30\%20No v\%2012.pdf.

[15] Sherwal, B.L., Gupta, P., Nayak, R., Gogoi, S., Suri, S. and Dutta, R. (2015) Prevalence of HIV in a Tertiary Care Centre in Delhi: A Five-Year ICTC Based Study. World Journal of AIDS, 5, 1-9. http://dx.doi.org/10.4236/wja.2015.51001

[16] Munawwar, A., Sharma, S.K., Gupta, S. and Singh, S. (2014) Seroprevalence and Determinants of Kaposi SarcomaAssociated Human Herpesvirus 8 in Indian HIV-Infected Males. AIDS Research and Human Retroviruses, 30, 11921196. http://dx.doi.org/10.1089/aid.2014.0184

[17] Huang, L., Cattamanchi, A., Davis, J.L., den Boon, S., Kovacs, J., Meshnick, S., et al. (2011) HIV-Associated Pneumocystis Pneumonia. Proceedings of the American Thoracic Society, 8, 294-300. http://dx.doi.org/10.1513/pats.201009-062WR 